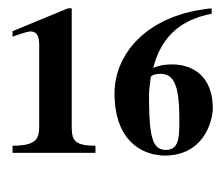

\title{
EL APRENDIZAJE DE LAS MUJERES SOBRE INTERNET Y REDES SOCIALES: VALIDACIÓN Y RESULTADOS GENERALES DE UNA ESCALA
}

(WOMEN'S LEARNING ON INTERNET AND SOCIAL NETWORKS: VALIDATION AND GENERAL RESULTS OF A SCALE)

\author{
Alba Vico-Bosch \\ Ángeles Rebollo-Catalán \\ Universidad de Sevilla
}

DOI: $10.5944 /$ educXX1.21469

\section{Cómo referenciar este artículo/How to reference this article:}

Vico-Bosch, A. y Rebollo-Catalán, Á. (2019). El aprendizaje de las mujeres sobre internet y redes sociales: validación y resultados generales de una escala. Educación $X X 1,22(1)$, 375-400, doi: 10.5944/educXX1.21469

Vico-Bosch, A. \& Rebollo-Catalán, Á. (2019). El aprendizaje de las mujeres sobre internet y redes sociales: validación y resultados generales de una escala. [Women's learning on internet and social networks: validation and general results of a scale]. Educación $X X 1,22(1)$, 375-400, doi: 10.5944/educXX1.21469

\section{RESUMEN}

Informes y políticas de inclusión digital a nivel internacional ponen el acento en la importancia de aumentar el protagonismo de las mujeres en la sociedad del conocimiento promoviendo sus capacidades en el uso de internet y las redes sociales. Este trabajo presenta tres escalas que sirven para medir su aprendizaje y algunos resultados preliminares obtenidos con las mismas. Aplicamos sendas escalas compuestas por 10 ítems cada una, que miden motivos, estrategias y recursos de aprendizaje respectivamente a 1.340 mujeres españolas de 18 a 65 años seleccionadas mediante un muestreo estratificado por cuotas considerando la edad y la situación laboral. Los resultados indican una óptima validez de constructo y un alto índice de fiabilidad de las tres escalas (motivos, $\alpha=.890$; estrategias, $\alpha=.900 ; \mathrm{y}$ recursos, $\alpha=.872$ ). Los resultados muestran que las mujeres aprenden a usar las redes sociales e internet por una amplia heterogeneidad de motivos, 
destacando a nivel específico la realización de gestiones administrativas, el desarrollo de hobbies o la gestión de su formación. También encontramos que las mujeres despliegan una variedad de estrategias usando más frecuentemente acciones de aprendizaje autónomo con apoyo en amigos y familiares, la consulta de webs y videotutoriales, observando cómo lo hacen otras personas y experimentando con la propia tecnología. En conclusión, las escalas se muestran valiosas para conocer cómo aprenden las mujeres sobre internet y las redes sociales. Este estudio abre el camino para repensar la oferta formativa encaminada a aumentar la visibilidad y protagonismo de las mujeres en la sociedad del conocimiento, considerando la naturaleza y diversidad del aprendizaje.

\section{PALABRAS CLAVE}

Redes sociales; aprendizaje; estudios de las mujeres; internet; fiabilidad; validación.

\section{ABSTRACT}

Reports and policies of digital inclusion on an international level highlight the importance of increasing the role of women in the knowledge society by promoting their skills in the use of the internet and social networks. This paper presents three scales used to measure their learning and some preliminary results obtained with them. We applied scales composed of 10 items each, which measure motives, strategies and learning resources respectively to 1,340 Spanish women aged 18 to 65, selected through stratified sampling by quotas considering age and employment status. The results indicate an optimal construct validity and a high reliability index of the three scales (motives, $\alpha=.890$, strategies, $\alpha=.900$, and resources, $\alpha=.872$ ). The results show that women learn to use social networks and the internet for a wide variety of reasons, highlighting at a specific level the performance of administrative procedures, the development of hobbies or the management of their training. We also find that women deploy a variety of strategies using more frequently autonomous learning actions with support from friends and family, consulting websites and videotutorials, observing how other people do it and experimenting with technology itself. In conclusion, the scales are valuable to know how women learn about the internet and social networks. This study opens the way to rethinking the training offer aimed at increasing the visibility and prominence of women in the knowledge society, considering the nature and diversity of learning.

\section{KEYWORDS}

Social networks sites; learning; women's studies; internet; reliability; validation. 


\section{INTRODUCCIÓN}

Esta investigación se plantea como respuesta a una necesidad manifestada durante la última década de forma reiterada en las políticas internacionales en materia de inclusión y alfabetización digital. Así como la brecha digital de género referida al acceso a Internet se ha venido reduciendo y, en especial, desde la popularización de la tecnología móvil, los estudios advierten de que la persistencia de las desigualdades de género en el uso de las tecnologías pueden perpetuarse en otras dimensiones como la experiencia, habilidad, autonomía y tipos de uso (Castaño, 2014; Hargittai, y Shafer, 2006; Hargittai, 2010). Algunas investigaciones recientes (Castaño, Duart y Sancho, 2012; Manosevitch y Tzuk, 2017; Nikitov y Sainty, 2014; Rebollo-Catalán, Mayor-Buzón, y García-Pérez, 2017) indican que son los usos profesionales junto con la creación de contenidos digitales lo que se asocia al logro de habilidades digitales avanzadas y es aquí donde la brecha de género se profundiza. Por ello, la Agenda Digital Europea (Comisión Europea, 2013) mantiene como línea prioritaria la mejora de la alfabetización digital, la capacitación e inclusión de las mujeres en la Sociedad de la Información. Y España, a través del Plan de Inclusión Digital y Empleabilidad (MIET, 2013), se fija como objetivo aumentar la presencia y participación de las mujeres en la Sociedad de la Información promoviendo acciones formativas dirigidas específicamente a mujeres que ya usan tecnologías digitales con el fin de aumentar sus capacidades. Esta investigación trata de aportar una herramienta válida y fiable, que arroje información útil para diseñar y adaptar las acciones formativas al perfil y necesidades expresadas por las mujeres.

Por otra parte, la irrupción de internet y las redes sociales han introducido importantes cambios en la educación para responder a las nuevas demandas de la sociedad, reconsiderándola en términos de una educación líquida y sin muros, donde el aprendizaje es móvil, conectado y distribuido (Castañeda y Adell, 2013; Gros, 2015; Tyner, Gutiérrez-Martín, y Torrego-González, 2015; Wheeler, 2015). Esto ha llevado a replantear el aprendizaje como un proceso amplio y dinámico que abarca diferentes contextos de actividad con oportunidades y recursos para aprender pero también ha cambiado la visión del aprendiz como persona activa, capaz de decidir qué, cómo, cuándo, dónde y con quién aprende (Coll y Engel, 2014; Sloep y Berlanga, 2011). Esto nos lleva a considerar la heterogeneidad de contextos, recursos, motivos y estrategias como dimensiones relevantes para comprender los procesos de aprendizaje en estos nuevos entornos virtuales.

Nuestra investigación se suma a otras que a nivel internacional están tratando de aportar escalas para medir nuevas formas de alfabetización surgidas a raíz del desarrollo tecnológico (Lau y Yuen, 2014; Lee, Chen, Li, 
y Lin, 2015). Sin embargo, la mayor parte de estudios previos se centran en la alfabetización mediática de poblaciones escolares en contextos muy diferentes al español. Pocos estudios se han centrado en las formas de aprendizaje surgidas con la aparición de las redes sociales virtuales e internet y aún menos en mujeres adultas.

En este contexto, la investigación que presentamos a continuación, financiada por el Programa de Investigación I+D de España, presenta y valida un instrumento de medida del aprendizaje en las redes sociales virtuales que puede servir para la evaluación diagnóstica del aprendizaje en estos entornos.

\section{ANTECEDENTES}

El aprendizaje y uso que hacen las mujeres de internet ha venido siendo un tema de interés desde hace ya largo tiempo (Alario y Anguita, 2001; Castaño, 2005; Jiménez-Cortés, 2004). Algunos de estos estudios han analizado los recursos y relaciones de apoyo que favorece el aprendizaje de las tecnologías (Prins, Toso, y Schafft, 2009; Rebollo-Catalán y Vico-Bosch, 2014). Así, Huber y Watson (2014) encuentran que los servicios y apoyo de un experto o la línea de ayuda son recursos en los que se apoyan las personas adultas en su aprendizaje relacionado con las tecnologías. Otros estudios descubren que es la ayuda de sus círculos íntimos de familiares y amigos el principal apoyo para su aprendizaje (Lin, Tang, y Kuo, 2012; Vega, VicoBosch, y Rebollo-Catalán, 2015). Por último, las investigaciones también señalan que las mujeres con mayor habilidad y autonomía en el uso de tecnologías prefieren usar foros, webs y videotutoriales como recursos para aprender (Sánchez-Vadillo, Orteg,a y Vall-Llovera, 2012; Vergés, Hache, y Cruells, 2011).

Paralelamente, algunas investigaciones recientes (Cobo y Moravec, 2011; Fernández-Rodríguez y Anguita, 2015; Greenhow y Lewin, 2016) han planteado la necesidad de integrar y tender puentes entre entornos de aprendizaje formal e informal como consecuencia de la aparición e impacto de las redes sociales e internet. En este sentido, encontramos que algunos trabajos han resaltado el papel del aprendizaje informal propiciados por las tecnologías digitales (Dabbagh y Kitsantas, 2012; Díez-Gutiérrez y DíazNafría, 2018; Heo y Lee, 2013; Jiménez-Cortés, 2015), mientras que otros (Coll y Engel, 2014; Rebollo-Catalán, Vico-Bosch, y García-Pérez, 2015) señalan que es la heterogeneidad y combinación de contextos y recursos, incluyendo las oportunidades ofrecidas por los entornos formales, la clave para entender el aprendizaje que permite a una persona alcanzar una ciudadanía plena en la sociedad 2.0. 
En relación con el aprendizaje en internet, las investigaciones también encuentran una variedad de estrategias de aprendizaje que usan las mujeres. De este modo, Vergés et al. (2011) encuentran que las mujeres con competencias tecnológicas avanzadas prefieren adoptar una estrategia autodidacta e informal, combinándola con la realización de cursos, talleres o jornadas de contenido muy específico. Por su parte, Rosenthal (2008) encuentra que, además de apoyarse en la ayuda informal de familiares, las mujeres mayores aprenden en cursos con el apoyo del profesorado pero también por ensayo y error. En su estudio sobre envejecimiento activo, Escarbajal, Martínez, y Salmerón (2015) encuentran que las mujeres mayores muestran una actitud más abierta que los hombres a nuevos aprendizajes, mostrando preferencia por las actividades de grupo. Otras investigaciones internacionales también observan la importancia de este tipo de actividades y contextos como una faceta clave para aprender en internet (Godfrey y Johnson, 2009; Lin et al., 2012).

La motivación de uso también ha sido analizada como un factor clave en el aprendizaje en redes sociales (Hargittai, 2010; Livingstone y Helsper, 2007). De este modo, aunque mantenerse en contacto con amigos y familiares y reducir la distancia geográfica son motivos generales que promueven el aprendizaje y uso de las redes sociales virtuales (Vega et al., 2015), también aparecen como motivaciones para aprender a usar las tecnologías su utilidad para resolver problemas de la vida cotidiana (Huber y Watson, 2014; Rubio y Escofet, 2013) o una vía para explorar aficiones y hobbies (Vergés et al., 2011). No obstante, la mayor parte de las investigaciones (Hargittai, 2010; Jiménez-Cortés, Rebollo-Catalán, García-Pérez, y Buzón-García, 2015) encuentran que es la heterogeneidad de motivos de uso lo que se asocia a mayores niveles de habilidad y autonomía de uso de las tecnologías, siendo las personas que mayor diversidad muestran en sus motivaciones para usar las redes sociales e internet, las que demuestran competencias avanzadas en su uso. En este trabajo, indagamos específicamente en los motivos de las mujeres para aprender el manejo y uso de las redes sociales virtuales.

En relación con el aprendizaje de internet, también encontramos algunos trabajos (Wastiau et al., 2013) que han reflexionado sobre los contenidos de la formación en tecnologías, identificando la necesidad de que esta tenga un fuerte componente práctico y que integre las áreas de interés y los contextos de aplicación. De este modo, algunos estudios documentan cómo las personas adultas aprenden a usar las redes sociales para informarse sobre salud, ocio y cultura (Agudo, Fombona, y Pascual, 2013; Casado-Muñoz, Lezcano y Rodríguez-Conde, 2015; FernándezCampomanes y Fueyo, 2014). 


\section{OBJETIVOS}

Con estos antecedentes, nuestro propósito principal en este trabajo es diseñar una escala válida, fiable y útil para medir los procesos de aprendizaje mediados por las redes sociales virtuales. De forma complementaria nos proponemos mostrar resultados obtenidos con la misma en una muestra de mujeres para conocer la forma en que aprenden el uso y aplicación de las redes sociales virtuales. De este modo podemos valorar la utilidad de la escala para indagar en las formas de aprendizaje de las redes sociales e internet.

\section{MÉTODO}

Para la realización de esta investigación aplicamos un diseño descriptivo correlacional basado en encuesta, por lo que se plantea con una metodología ex post facto que permite explorar la naturaleza y estructura del fenómeno objeto de estudio.

\section{Participantes}

La población objeto de estudio, según datos facilitados por el Instituto Nacional de Estadística de España, está constituida por 12.150.042 mujeres españolas adultas que usan internet y redes sociales. La muestra está formada por 1.340 mujeres españolas, seleccionadas mediante un muestreo estratificado por cuotas, considerando edad y situación laboral. Estaríamos ante un tamaño muestral estadísticamente representativo para un nivel de confianza del 95\%, un error inferior al $\pm 3 \%$ y $\mathrm{P}=\mathrm{Q}$. Contemplamos como único criterio de inclusión que estas tuvieran al menos un año de experiencia en el uso de redes sociales virtuales.

La muestra está compuesta por 24,1\% de mujeres de 18 a 25 años, 22,3\% de 45 a 54 años, 21,2\% de 35 a 44 años, 16,3\% de 26 a 34 años y $16,1 \%$ mayor de 55 años. La mayoría tiene estudios secundarios $(37,8 \%)$ o universitarios (34,9\%), siendo el $27,3 \%$ de mujeres las que tienen estudios primarios. En cuanto a la situación laboral, la mayoría de las mujeres son profesionales $(45,8 \%)$ o desempleadas $(42,5 \%)$, siendo solo un $11,7 \%$ empresarias.

En cuanto al perfil tecnológico de las mujeres, encontramos que más del $80 \%$ de las mujeres usan dispositivos móviles para manejar las redes sociales, siendo el móvil y el portátil los más usados. El 86,3\% de ellas lo tiene a su disposición de forma exclusiva. Las redes que más usan son 
WhatsApp, Facebook y YouTube, siendo un 50,3\% las mujeres que las usan de forma combinada.

\section{Variables e instrumento}

Partiendo de algunas aportaciones previas (Greenhow y Robelia, 2009; Gros, 2015; Jiménez-Cortés, 2015), definimos nuestra variable de estudio como «un proceso de adquisición de conocimientos y habilidades en el uso de internet y de las redes sociales, que emprenden las personas impulsadas por determinados motivos para lo cual emplean una variedad de estrategias y recursos a través de un amplio rango de contextos».

El instrumento empleado para llevar a cabo la investigación es el cuestionario, en el que cada ítem adopta un rango de respuesta de 4 puntos donde 0 es nunca, 1 rara vez, 2 algunas veces y 3 muchas veces. Dicho cuestionario incluye 3 escalas que miden:

1. Motivos: que mide los propósitos de las personas para aprender en y sobre redes sociales virtuales e internet (Jiménez-Cortés et al., 2015; Livingstone y Helsper, 2007).

2. Estrategias: escala que mide la predisposición de las personas al uso de distintas formas de aprendizaje en internet, las cuales van desde fórmulas más convencionales basadas en la realización de cursos hasta estrategias de carácter más autodidacta (Rosenthal, 2008; Vergés et al., 2011).

3. Recursos: escala que recoge los entornos y recursos que emplean las personas para aprender a usar las redes sociales e internet (Greenhow y Robelia, 2009; Huber y Watson, 2014).

\section{Procedimiento}

Una vez determinado el tamaño de la muestra, se selecciona la muestra mediante un muestreo estratificado por cuotas. Para el proceso de recogida de la información contamos con la colaboración del Instituto Andaluz de la Mujer y otras organizaciones de mujeres que nos facilitaron el acceso a la muestra. Las encuestadoras administraron el cuestionario en persona en las dependencias correspondientes a salas de informática de los diferentes municipios. Para facilitar la cumplimentación por parte de la muestra y también la posterior extracción de los datos, diseñamos la encuesta en formato electrónico a través de Google Form, incluyendo una 
sección con información sobre la finalidad y usos de la investigación y sobre la naturaleza anónima de las respuestas, obteniendo el consentimiento y autorización de las participantes para usar la información de forma electrónica. La recogida de datos se realiza entre los meses de abril y septiembre de 2015.

Los datos obtenidos fueron analizados mediante el paquete estadístico SPSS (versión 22). Realizamos un análisis de validez y fiabilidad de las medidas. Para el análisis de fiabilidad aplicamos el coeficiente $\alpha$ de Cronbach tanto de la escala global como de las subescalas que la componen. Para la validez de constructo, aplicamos un análisis factorial exploratorio de componentes principales para cada escala.

Para este trabajo presentamos los resultados en una escala de tres puntos (rara vez, algunas veces, muchas veces), una vez comprobada que dicha recodificación no afecta a su validez y fiabilidad. Para extraer resultados realizamos análisis descriptivos básicos (medidas de tendencia central, dispersión y distribución) para conocer el comportamiento de las variables en la muestra.

\section{RESULTADOS}

\section{Validez de constructo}

Realizamos un análisis factorial exploratorio (AFE) de las escalas de motivos, estrategias y recursos de aprendizaje respectivamente, para estudiar su estructura y composición de la medida del aprendizaje tecnológico de las mujeres.

\section{Validez de la escala motivos de aprendizaje}

Se aplica un análisis factorial de componentes principales de máxima verosimilitud con rotación Varimax a la medida de motivos de aprendizaje de las redes sociales que arroja una solución en dos factores que explica el $51,28 \%$ de la varianza total (26,61\% el factor $1 ; 24,67 \%$ el factor 2$)$. El análisis de la fiabilidad indica un alpha de Cronbach .83 para el factor 1 (usos generales) y .82 para el factor 2 (usos específicos). Todos los ítems obtuvieron pesos factoriales superiores a .30 en el factor al que fueron asignados (tabla 1), tomando como criterio asignar el ítem al factor donde su peso fuera mayor. 
Tabla 1

Matriz de componentes rotados motivos de aprendizaje

\begin{tabular}{|c|c|c|}
\hline $\begin{array}{c}\text { (Varianza total explicada: } 51,28 \% ; \mathrm{KMO}=0,895 \\
\text { Prueba de esfericidad de Barlett: } \\
\text { Chi-cuadrado = 5515,719; gl = 45; Sig. }=, 000)\end{array}$ & \multirow[t]{2}{*}{$\begin{array}{l}\text { Factor } 1 \\
\text { Usos } \\
\text { generales }\end{array}$} & \multirow[t]{2}{*}{$\begin{array}{l}\text { Factor } 2 \\
\text { Usos } \\
\text { específicos }\end{array}$} \\
\hline Ítems & & \\
\hline $\begin{array}{l}\text { ÍTEM 2. Relacionarme con personas de diversas edades, } \\
\text { culturas, etc. }\end{array}$ & ,827 & \\
\hline ÍTEM 1. Relacionarme y expresarme más abiertamente & ,727 & \\
\hline $\begin{array}{l}\text { ÍTEM 4. Participar más activamente en la vida cultural } \\
\text { y social de mi comunidad }\end{array}$ & ,655 & ,351 \\
\hline $\begin{array}{l}\text { ÍTEM 3. Reducir la distancia geográfica con personas, } \\
\text { grupos y comunidades }\end{array}$ & ,625 & \\
\hline $\begin{array}{l}\text { ÍTEM 5. Atender mejor mis necesidades y las de mi fa- } \\
\text { milia }\end{array}$ & ,495 & ,447 \\
\hline ÍTEM 6. Ser más eficiente y productiva en mi trabajo & & ,780 \\
\hline $\begin{array}{l}\text { ÍTEM 8. Gestionar mejor mis actividades de formación, } \\
\text { mis estudios }\end{array}$ & & ,755 \\
\hline $\begin{array}{l}\text { ÍTEM 9. Realizar gestiones administrativas (operaciones } \\
\text { bancarias, compra online...) }\end{array}$ & &, 549 \\
\hline $\begin{array}{l}\text { ÍTEM 7. Experimentar y desarrollar mis hobbies (pin- } \\
\text { tura, cocina, viajes...) }\end{array}$ & ,411 &, 540 \\
\hline ÍTEM 10. Promocionar y dar visibilidad a mi empresa & & ,421 \\
\hline \multicolumn{3}{|l|}{$\begin{array}{l}\text { Método de extracción: análisis de componentes principales. } \\
\text { Método de rotación: normalización varimax Kaiser. } \\
\text { La rotación ha convergido en } 3 \text { iteraciones. }\end{array}$} \\
\hline
\end{tabular}

\section{Validez de la escala estrategias de aprendizaje}

Se aplica un análisis factorial de componentes principales de máxima verosimilitud con rotación oblimin a la medida de las estrategias de aprendizaje, que arroja una solución en tres factores que explica el 64,95\% de la varianza total (46,95\% el factor 1; 14,4\% el factor 2; 3,60\% el factor 3). El análisis de la fiabilidad indica un alpha de Cronbach .85 para el factor 1 (convencional), .83 para el factor 2 (autónoma) y .84 para el factor 3 (colaborativa). Todos los ítems obtuvieron pesos factoriales superiores a .30 en el factor al que fueron asignados (tabla 2), tomando como criterio asignar el ítem al factor donde su peso fuera mayor. La eliminación del 
ítem 7 garantiza la validez de contenido, a la vez que aumenta la varianza explicada.

Tabla 2

Matriz de componentes rotados estrategias de aprendizaje

\begin{tabular}{|c|c|c|c|}
\hline $\begin{array}{l}\text { (Varianza total explicada: } 65,41 \% \text {; } \\
\text { KMO = 0,875; } \\
\text { Prueba de esfericidad de Barlett: } \\
\text { Chi-cuadrado }=4147,069 ; \text { gl = } 36 ; \\
\text { Sig. }=, 000)\end{array}$ & $\begin{array}{c}\text { Factor } 1 \\
\text { Convencional }\end{array}$ & $\begin{array}{c}\text { Factor } 2 \\
\text { Autónoma }\end{array}$ & $\begin{array}{c}\text { Factor } 3 \\
\text { Colaborativa }\end{array}$ \\
\hline \multicolumn{4}{|l|}{ Ítems } \\
\hline $\begin{array}{l}\text { ÍTEM 5. Asistiendo a cursos sobre he- } \\
\text { rramientas y aplicaciones específicas } \\
\text { y útiles }\end{array}$ & ,933 & & ,594 \\
\hline $\begin{array}{l}\text { ÍTEM 8. Asistiendo a cursos sobre } \\
\text { cómo funcionan a nivel básico para } \\
\text { después seguir aprendiendo yo sola }\end{array}$ & 831 & & 648 \\
\hline $\begin{array}{l}\text { ÍTEM 4. Realizando cursos virtuales } \\
\text { que me permiten ir aplicando lo que } \\
\text { aprendo }\end{array}$ & 658 & & 653 \\
\hline $\begin{array}{l}\text { ÍTEM 2. Indagando y probando mien- } \\
\text { tras intento hacer cosas que me gus- } \\
\text { tan }\end{array}$ & & ,836 & ,524 \\
\hline $\begin{array}{l}\text { ÍTEM 3. Usándola de forma intuitiva } \\
\text { por el placer de experimentar con la } \\
\text { tecnología }\end{array}$ & & ,826 & 491 \\
\hline $\begin{array}{l}\text { ÍTEM 6. Formando parte de un grupo } \\
\text { o comunidad que me hace sentir su } \\
\text { apoyo }\end{array}$ & 630 & & 808 \\
\hline $\begin{array}{l}\text { ÍTEM 10. Apuntándome a actividades } \\
\text { organizadas por asociaciones y gru- } \\
\text { pos que conozco }\end{array}$ & ,740 & & ,741 \\
\hline $\begin{array}{l}\text { ÍTEM 9. Dialogando con otras perso- } \\
\text { nas en foros y grupos }\end{array}$ & 402 & ,531 & ,705 \\
\hline $\begin{array}{l}\text { ÍTEM 1. Participando con otras per- } \\
\text { sonas en proyectos comunes }\end{array}$ & 433 & ,486 & 690 \\
\hline $\begin{array}{l}\text { Método de extracción: análisis de com } \\
\text { Método de rotación: normalización ob } \\
\text { La rotación ha convergido en } 11 \text { iterac }\end{array}$ & $\begin{array}{l}\text { onentes princ } \\
\text { min con Kais } \\
\text { ones. }\end{array}$ & & \\
\hline
\end{tabular}




\section{Validez de la escala recursos de aprendizaje}

Se aplica un análisis factorial de componentes principales de máxima verosimilitud con rotación Varimax a la medida de recursos de aprendizaje, adoptando una solución en tres factores que explica el 55,32\% de la varianza total (22,49\% el factor $1 ; 18,75 \%$ el factor 2; $14,08 \%$ el factor 3 ). El análisis de la fiabilidad indica un alpha de Cronbach .86 para el factor 1 (informal-autónomo), .85 para el factor 2 (formal-experto) y .67 para el factor 3 (informal-social). Como puede observarse (tabla 3) todos los ítems obtuvieron pesos factoriales superiores a .30 en el factor al que fueron asignados, para lo cual se tomó como criterio asignar el ítem al factor donde su peso fuera mayor. Se suprimió el ítem 8 para asegurar la validez de contenido, maximizar la diferenciación de factores y aumentar la varianza explicada.

Tabla 3

Matriz de componentes rotados recursos de aprendizaje

\begin{tabular}{|c|c|c|c|}
\hline $\begin{array}{c}\text { (Varianza total explicada: } 55,32 \% ; \text { KMO }=0,760 ; \\
\text { Prueba de esfericidad de Barlett: } \\
\text { Chi-cuadrado = 2753,797; gl = 36; Sig. }=, 000)\end{array}$ & \multirow{2}{*}{$\begin{array}{l}\text { Factor } 1 \\
\text { Informal- } \\
\text { autónomo }\end{array}$} & \multirow{2}{*}{$\begin{array}{c}\text { Factor } 2 \\
\text { Formal- } \\
\text { experto }\end{array}$} & \multirow{2}{*}{$\begin{array}{l}\text { Factor } 3 \\
\text { Informal- } \\
\quad \text { social }\end{array}$} \\
\hline Ítems & & & \\
\hline $\begin{array}{l}\text { ÍTEM 4. Consultando información por Internet } \\
\text { (buscadores, blogs...) }\end{array}$ & ,865 & & \\
\hline $\begin{array}{l}\text { ÍTEM 5. Buscando vídeos tutoriales por You- } \\
\text { Tube }\end{array}$ & ,838 & & \\
\hline $\begin{array}{l}\text { ÍTEM 6. Experimentando y probando con la } \\
\text { propia tecnología }\end{array}$ & ,640 & & \\
\hline ÍTEM 9. Realizando cursos virtuales & & ,757 & \\
\hline $\begin{array}{l}\text { ÍTEM 7. Asistiendo a cursos, talleres y otras ac- } \\
\text { tividades formativas presenciales }\end{array}$ & & ,705 & \\
\hline ÍTEM 1. Llamando a la línea de ayuda & & 492 & \\
\hline $\begin{array}{l}\text { ÍTEM 3. Contratando los servicios de un ex- } \\
\text { perto }\end{array}$ & & 475, & \\
\hline $\begin{array}{l}\text { ÍTEM 10. Observando cómo lo hacen otras per- } \\
\text { sonas de mi entorno }\end{array}$ & & & ,962 \\
\hline ÍTEM 2. Pidiendo ayuda a amigos y familiares & & & ,438 \\
\hline \multicolumn{4}{|c|}{$\begin{array}{l}\text { Método de extracción: análisis de componentes principales. } \\
\text { Método de rotación: normalización Varimax Kaiser. } \\
\text { La rotación ha convergido en } 5 \text { iteraciones. }\end{array}$} \\
\hline
\end{tabular}




\section{Análisis de fiabilidad}

Los valores de consistencia interna tanto de la escala global como de las tres escalas son aceptables como refleja la tabla 4. De esta forma, podemos afirmar que tanto la escala global como las tres escalas que incluye poseen índices de fiabilidad o consistencia interna altos al alcanzar coeficientes alpha de Cronbach que están por encima de .88.

Tabla 4

Análisis de fiabilidad

\begin{tabular}{lcc}
\hline \multicolumn{1}{c}{ Consistencia interna ( $\boldsymbol{\alpha}$ de Cronbach) } & Valores & $\begin{array}{c}\mathbf{N}^{\circ}{ }^{\circ} \text { de } \\
\text { ítems }\end{array}$ \\
\hline Escala global & .943 & 28 \\
Motivos de aprendizaje & .890 & 10 \\
Estrategias de aprendizaje & .900 & 9 \\
Recursos de aprendizaje & .872 & 9 \\
\hline
\end{tabular}

\section{Resultados globales}

Los datos indican que los motivos de aprendizaje de las mujeres se refieren fundamentalmente a usos generales, acudiendo a estos un 53,6\% de forma ocasional y un 27,4\%, de forma frecuente. En contraste, solo el 19,1\% hace usos específicos de las redes con frecuencia para aprender (tabla 5).

Tabla 5

Frecuencia de motivos de aprendizaje

\begin{tabular}{lcc}
\hline & $\begin{array}{c}\text { Usos generales } \\
(\mathbf{n = 1 3 1 6 )}\end{array}$ & $\begin{array}{c}\text { Usos } \\
\text { específicos } \\
(\mathbf{n = 1 2 9 0 )}\end{array}$ \\
\hline Rara vez & $250(19,0 \%)$ & $415(32,2 \%)$ \\
Algunas veces & $705(53,6 \%)$ & $628(48,7 \%)$ \\
Muchas veces & $361(27,4 \%)$ & $247(19,1 \%)$ \\
\hline
\end{tabular}

Como podemos comprobar en la figura 1, entre los motivos de carácter general destaca de manera ocasional y frecuente reducir distancia geográfica $(77,7 \%)$ y, entre los motivos más específicos, realizar gestiones administrativas $(58,7 \%)$, desarrollar hobbies $(60,7 \%)$ o gestionar mejor la formación $(58,2 \%)$. 


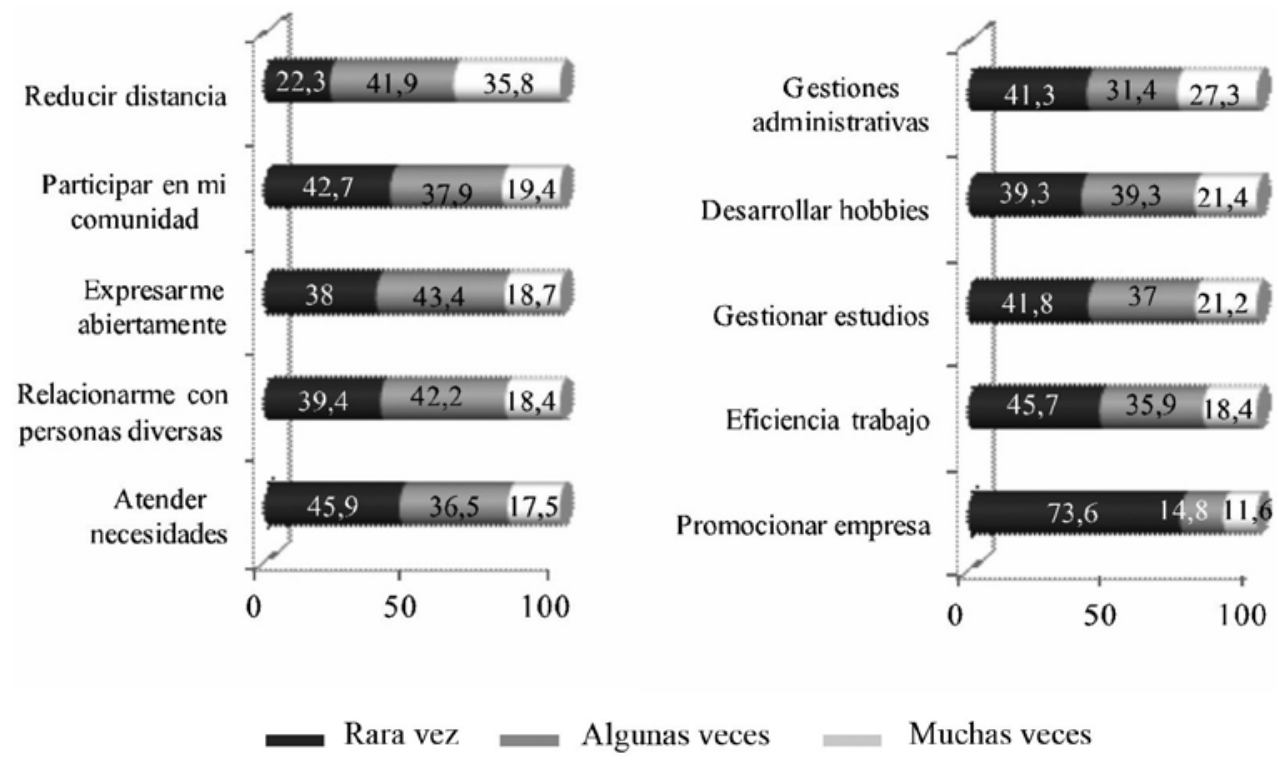

Figura 1. Distribución de motivos de aprendizaje

En cuanto a las estrategias de aprendizaje, las mujeres indican que la forma de aprender el uso de redes sociales más frecuente es la autodidacta (51,9\%), acudiendo a fórmulas de aprendizaje colaborativo y de formación convencional basada en cursos de forma ocasional con un 66,6\% y 48,4\% respectivamente (tabla 6).

Tabla 6

Frecuencia de estrategias de aprendizaje

\begin{tabular}{lccc}
\hline & $\begin{array}{c}\text { Autónomo } \\
(\mathbf{n = 8 6 1})\end{array}$ & $\begin{array}{c}\text { Convencional } \\
(\mathbf{n = 8 5 8})\end{array}$ & $\begin{array}{c}\text { Colaborativo } \\
(\mathbf{n = 8 5 1})\end{array}$ \\
\hline Rara vez & $70(8,1 \%)$ & $217(25,3 \%)$ & $105(12,3 \%)$ \\
Algunas veces & $344(40,0 \%)$ & $415(48,4 \%)$ & $567(66,6 \%)$ \\
Muchas veces & $447(51,9 \%)$ & $226(26,3 \%)$ & $179(21,0 \%)$ \\
\hline
\end{tabular}

La figura 2 refleja la diversidad en las estrategias que emplean las mujeres para aprender en estos entornos. Como estrategia autodidacta un $64,3 \%$ indica de forma ocasional o frecuente indagar y probar con la tecnología mientras realizan cosas que les gustan. Pero también y, de manera frecuente, un $15 \%$ realiza cursos sobre aplicaciones específicas y un $14,2 \%$ cursos básicos para después seguir aprendiendo sola. Con respecto a 
estrategias colaborativas un 14,2\% de las mujeres aprende participando con otras personas en proyectos comunes.

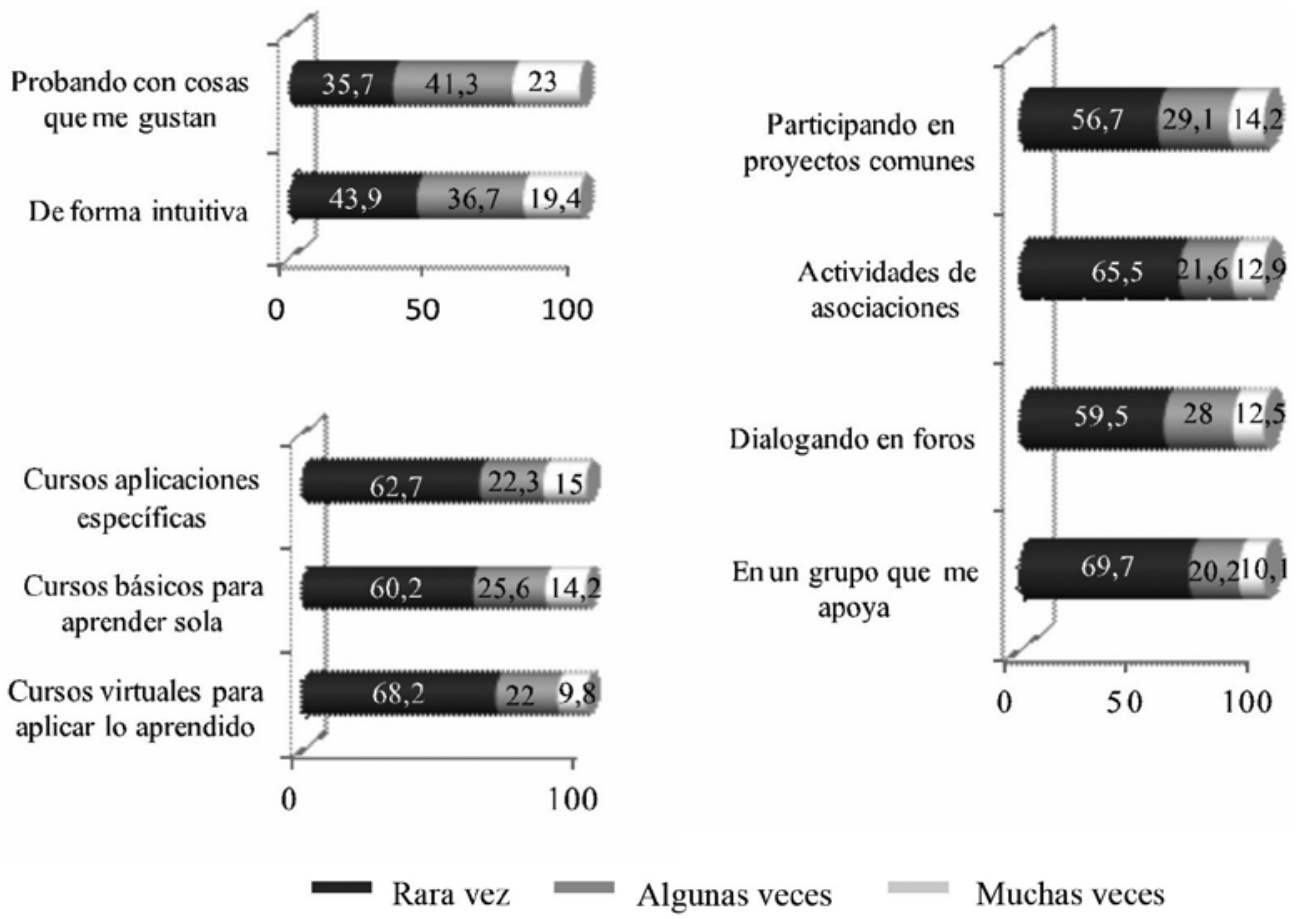

Figura 2. Distribución de estrategias de aprendizaje

Por último, con respecto a los contextos y recursos de aprendizaje la tabla 7 nos muestra que las mujeres prefieren hacerlo en contextos informales bien con apoyo social $(61,8 \%)$ bien experimentando de forma autónoma con la propia tecnología $(48,2 \%)$. Los contextos formales con apoyo en expertos se usa de forma más puntual $(8,5 \%)$.

Tabla 7

Frecuencia de recursos de aprendizaje

\begin{tabular}{lccc}
\hline & $\begin{array}{c}\text { Informal-social } \\
(\mathbf{n}=\mathbf{8 6 5})\end{array}$ & $\begin{array}{c}\text { Informal-autónomo } \\
\mathbf{( n = 8 6 7 )}\end{array}$ & $\begin{array}{c}\text { Formal-experto } \\
(\mathbf{n}=\mathbf{8 6 2})\end{array}$ \\
\hline Rara vez & $29(3,4 \%)$ & $65(7,5 \%)$ & $207(24,0 \%)$ \\
Algunas veces & $301(34,8 \%)$ & $384(44,3 \%)$ & $582(67,5 \%)$ \\
Muchas veces & $535(61,8 \%)$ & $418(48,2 \%)$ & $73(8,5 \%)$ \\
\hline
\end{tabular}


Los resultados muestran que las mujeres se apoyan en su entorno más cercano con la ayuda de amigos y familiares $(41,1 \%)$, la consulta de webs (26,2\%), de videotutoriales (24\%), observando a otros $(22,7 \%)$ y experimentando con la propia tecnología $(22,4 \%)$. Acuden con menos frecuencia a la ayuda de expertos mediante cursos presenciales o virtuales como recurso para aprender (10,3\%) (figura 3$)$.

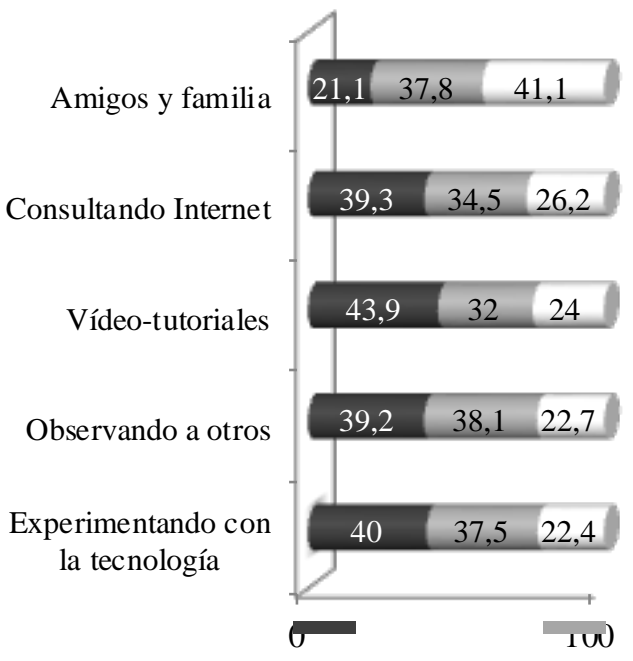

Rara vez

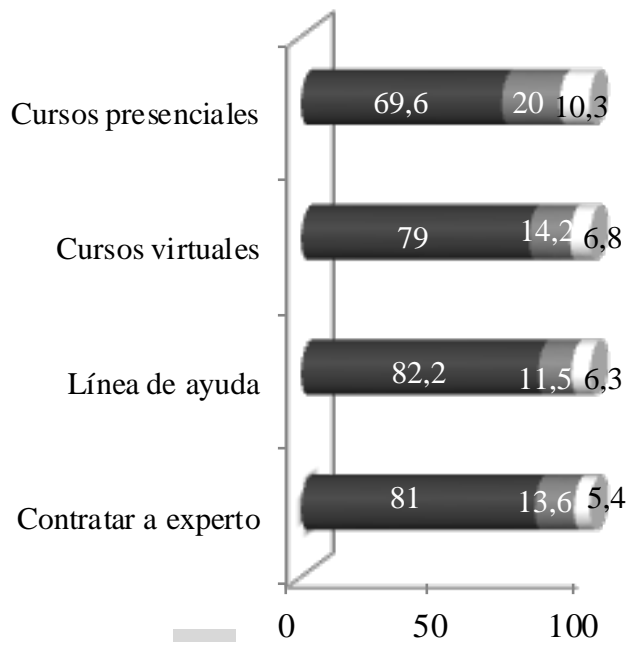

Muchas veces

Figura 3. Distribución de recursos de aprendizaje

\section{DISCUSIÓN Y CONCLUSIONES}

Con este trabajo hemos querido aportar una herramienta valiosa y útil para medir procesos de aprendizaje de las mujeres en redes sociales virtuales e internet. Nuestro propósito ha sido proporcionar una medida que permita generar conocimiento útil y aplicado sobre su aprendizaje, centrándonos en los cambios que han introducido estos espacios digitales en el aprendizaje de las mujeres. Aunque se ha escrito y reflexionado sobre el aprendizaje en estos entornos (Anderson, 2010; Wheeler, 2015), pocos trabajos han aportado medidas sobre los procesos de aprendizaje en estos espacios. Con este estudio, hemos querido proporcionar una herramienta 
útil para el diagnóstico de necesidades que sirva para adaptar y personalizar las acciones formativas que se desarrollan en el marco de planes y programas orientados a la inclusión digital.

Sin menoscabo de otras dimensiones que puedan ser igualmente relevantes para el estudio del aprendizaje en redes sociales virtuales, con este trabajo documentamos la relevancia de los motivos, estrategias y recursos como dimensiones clave en el aprendizaje de TIC. Estas dimensiones aparecen de forma reiterada en la literatura científica reciente (Castañeda y Adell, 2013; Meyers, Erikson, y Small, 2013; Sánchez-Vadillo et al., 2012).

Tomando como punto de partida los estudios más recientes (Cobo y Moravec, 2011; Coll y Engel, 2014; Fernández-Rodríguez y Anguita, 2015), la escala sobre recursos de aprendizaje que hemos diseñado se muestra sensible para medir la participación no solo de distintos contextos en los procesos de aprendizaje en TIC sino también de los recursos sobre los que se sostiene el aprendizaje en estos. De este modo, la escala no solo incluye ítems relacionados con el contexto formal e informal, sino que muestra los recursos en los que se apoya el aprendizaje, identificando la ayuda de personas expertas, del círculo más íntimo de familia y amigos o de la propia tecnología a través de videotutoriales, webs, etc. como opciones reconocibles en el proceso de aprendizaje. El análisis factorial muestra una estructura en tres dimensiones en la que se distingue la presencia de un contexto informal más inmediato basado en el apoyo de personas del entorno social más cercano de familiares y amigos, un contexto informal más ampliado basado en el apoyo de los propios recursos tecnológicos (videotutoriales, webs, etc.) y un contexto formal con apoyo de personas expertas. Estos resultados concuerdan con los de investigaciones previas en las que se demuestra la participación de contextos formales e informales en el aprendizaje (Dabbagh y Kitsantas, 2012; Heo y Lee, 2013; JiménezCortés, 2015; Rebollo-Catalán et al., 2015) así como los de aquellas que han documentado los diversos recursos que utilizan las personas para apoyar su aprendizaje (Huber y Watson, 2014; Lin et al., 2012; Sánchez-Vadillo et al., 2012; Vergés et al., 2011).

Con este artículo también mostramos una escala sobre los motivos que impulsan a las mujeres a aprender a usar las redes sociales, identificando usos generales como reducir la distancia geográfica, participar activamente en su comunidad y relacionarse con personas muy diversas, ya presentes en investigaciones previas, y motivaciones vinculadas a usos específicos asociados al desarrollo personal, cultural y profesional. Aunque existe abundante bibliografía previa sobre esta dimensión con aportación de medidas (Hew y Cheung, 2012; Yang y Brown, 2013), la mayor parte han sido realizadas con listas de control. Nuestro estudio no solo permite registrar las 
motivaciones presentes en el acercamiento a las redes sociales, sino también el grado en que esas motivaciones han impulsado el aprendizaje de las redes sociales. Por otra parte, como las investigaciones previas han mostrado (Hargittai, 2010; Livingstone y Helsper, 2007), es la heterogeneidad de motivos lo que incide en la alfabetización digital y no el tipo de motivación, habiendo documentado que son las personas que combinan diez o más motivos, las que muestran un mayor nivel de habilidad y autonomía digital. Con nuestra escala exploramos esa variedad siendo, a la vez, una escala viable y fácil de aplicar.

Nuestro estudio también aporta una escala para medir las estrategias que emplean las personas en su aprendizaje de las redes sociales, encontrando que junto con modalidades más convencionales basadas en la realización de cursos, nuestra escala también recoge fórmulas basadas en el aprendizaje colaborativo y autónomo. Los resultados obtenidos con esta escala pueden servir para adecuar las estrategias formativas más idóneas para favorecer el aprendizaje en entornos digitales.

Los resultados preliminares de aplicación de estas escalas nos arrojan información de interés sobre la forma en que aprenden las mujeres sobre internet y redes sociales, siendo lo más significativo el uso de estrategias autodidactas en contextos informales y con apoyo en la ayuda de su entorno más inmediato de amigos y familiares o de la propia tecnología consultando información por internet o videotutoriales. La realización de cursos y la ayuda de personas expertas es una opción empleada de forma ocasional y puntual. Investigaciones previas realizadas con metodologías cualitativas también obtienen resultados similares (Sánchez-Vadillo et al., 2012; Vega et al., 2015; Vergés et al., 2011).

Esta investigación abre la puerta a la realización de futuros estudios sobre nuevos perfiles y formas de aprendizaje que permita repensar la función de la educación formal y su relación con los contextos informales de aprendizaje. Estas investigaciones pueden aportar las evidencias necesarias para flexibilizar, diversificar y adecuar la formación proporcionada en contextos formales a las necesidades y preferencias de aprendizaje de distintos colectivos y grupos tal y como vienen planteando diversos autores (Coll y Engel, 2014; Fernández-Rodríguez y Anguita, 2015; Tyner et al., 2015; Díez-Gutiérrez y Díaz-Nafría, 2018) que abogan por una educación formal más abierta y flexible que tenga en cuenta los aprendizajes de las personas en distintos contextos de actividad. Aportar evidencias sobre la heterogeneidad de las formas de aprendizaje mediadas por las redes sociales virtuales se hace necesario, siendo preciso disponer de una variedad de herramientas metodológicas que resulten útiles para hacerlo. 
Antes de finalizar queremos señalar algunas limitaciones de este estudio que pueden condicionar el uso y aplicación de la escala que presentamos. La primera limitación deriva del tipo de medida, ya que al estar basada en un cuestionario de autoinforme sería aconsejable introducir medidas complementarias de validez concurrente para dar mayor robustez a la evidencia empírica. La segunda limitación deriva de la naturaleza de la muestra elegida, ya que al estar constituida solo por mujeres adultas, la validez de la medida se restringe exclusivamente a este colectivo, siendo obligatorio en caso de su aplicación en otros grupos validar nuevamente la medida mediante estudios pilotos para valorar la adecuación de su aplicación con un carácter más amplio. Por último, queremos señalar la limitación derivada del enfoque metodológico adoptado. Creemos que el uso de métodos mixtos complementados con metodologías cualitativas a este estudio cuantitativo permitiría contrastar y triangular la información obtenida.

\section{NOTAS}

1 Este trabajo se ha podido realizar gracias a la concesión del proyecto de investigación $\mathrm{I}+\mathrm{D}$ «Las mujeres como tejedoras de las redes sociales: estrategias relacionales e inclusión digital» (EDU2013-45134-P), financiado por el Ministerio de Economía y Competitividad.

2 Este trabajo presenta resultados parciales de la tesis doctoral de Alba Vico Bosch, financiada por el Ministerio de Educación, Cultura y Deporte de España (FPU13/03826) y desarrollada en el marco de este proyecto. 


\section{REFERENCIAS BIBLIOGRÁFICAS}

Agudo, S., Fombona, J., y Pascual, M.A. (2013). Ventajas de la incorporación de las TIC en el envejecimiento. RELATEC, 12(2), 131-142.

Alario, A.I., y Anguita, R. (2001). Las mujeres, las nuevas tecnologías y la educación, un camino lleno de obstáculos. En M. Area (Coord.), Educar en la Sociedad de la Información (pp. 215-247). Bilbao: Desclée de Brouwer.

Anderson, T. (2010). Theories for learning with emerging technologies. En G. Valesianos (Ed.), Emerging technologies in distance education (pp. 23-40). Edmonton, Canada: AU Press, Athabasca University.

Casado-Muñoz, R., Lezcano, F., y Rodríguez-Conde, M.J. (2015). Envejecimiento activo y acceso a las tecnologías: Un estudio empírico evolutivo. Comunicar, 45, 37-46. doi: 10.3916/C45-2015-04

Castañeda, L., y Adell, J. (Eds.) (2013). Entornos Personales de Aprendizaje: claves para el ecosistema educativo en red. Alcoy: Marfil.

Castaño, C. (2005). Las mujeres y las tecnologías de la información. Internet y la trama de nuestra vida. Madrid: Alianza.

Castaño, C. (2014). Women's Access to ICTs in the information society. En A. Vega-Montiel (Ed.), Media and Gender: a Scholarly Agenda for the Global Alliance on Media and Gender (pp. 6065). Francia: UNESCO.

Castaño, J., Duart J.M., y Sancho, T. (2012). Una segunda brecha digital entre el alumnado universitario. Culturay Educación, 24(3), 363-377.
Cobo, C., y Moravec, J.W. (2011). Aprendizaje invisible. Hacia una nueva ecología de la educación. Barcelona: Laboratori de Mitjans Interactius / Publicacions i Edicions de la Universitat de Barcelona.

Coll, C., y Engel, A. (2014). Introduction: Personal Learning Environments in the context of formal education. Cultura y Educación, 26(4), 617-630. doi: 10.1080/11356405.2014.985947

Comisión Europea (2013). Europa 2020: la estrategia europea de crecimiento. Recuperado de https://goo.gl/DvY8wb

Dabbagh, N., \& Kitsantas, A. (2012). Personal Learning Environments, social media and self-regulated learning: A natural formula for connecting formal and informal learning. The Internet \& Higher Education, 15(1), 3-8. doi: 10.1016/j. iheduc.2011.06.002

Díez-Gutiérrez, E., y Díaz-Nafría, J.M. (2018). Ecologías de aprendizaje ubicuo para la ciberciudadanía crítica. Comunicar, 54, 49-58. doi: 10.3916/ C54-2018-05

Escarbajal, A., Martínez, S.M., y Salmerón, J.A. (2015). La percepción de la calidad de vida en las mujeres mayores y su envejecimiento activo a través de actividades socioeducativas en los centros sociales. Revista de Investigación Educativa, 33(2), 471488. doi: 10.6018/rie.33.2.213211

Fernández-Campomanes, M., y Fueyo, A. (2014). Redes sociales y mujeres mayores: Estudio sobre la influencia de las redes sociales en la calidad de vida. Revista Mediterránea de Comunicación, 5(1), 157-177. doi: 10.14198/MEDCOM2014.5.1.11 
Fernández-Rodríguez, E., y Anguita, R. (2015). Aprendizajes invisibles en contextos de educación expandida. Retos y oportunidades en la sociedad hiperconectada. Profesorado, 19(2), $1-16$.

Godfrey, M., \& Johnson, O. (2009). Digital circles of support: Meeting the information needs of older people. Computers in Human Behavior, 25(3), 633-642. doi: 10.1016/j. chb.2008.08.016

Greenhow, C., \& Lewin, C. (2016). Social media and education: reconceptualizing the boundaries of formal and informal learning. Learning, Media and Technology, 41(1),6-30.doi: 10.1080/17439884.2015.1064954

Greenhow, C., \& Robelia, B. (2009). Informal learning and identity formation in online social networks.Learning, Media and Technology, 34(2), 119-140. doi: 10.1080/17439880902923580

Gros, B. (2015). La caída de los muros del conocimiento en la sociedad digital y las pedagogías emergentes. Education in the Knowledge Society, 16(1), 58-68.

Hargittai, E., \& Shafer, S. (2006). Differences in actual and perceived online skills: The role of gender. Social Science Quarterly, 87(2), 432-448. doi: 10.1111/j.1540-6237.2006.00389.x

Hargittai, E. (2010). Digital Natives? Variation in Internet Skills and Uses among Members of the "Net Generation', Sociological Inquiry, 80(1), 92-113. doi: 10.1111/j.1475682X.2009.00317.x

Heo, G.M., \& Lee, R. (2013). Blogs and social networks sites as activity systems: exploring adult informal learning process through activity theory framework. Educational Technology \& Society, 16(4), 133-145.

Hew, K.F., \& Cheung, W.S. (2012). Students' use of asynchronous voice discussion in a blendedlearning environment: A study of two undergraduate classes. The Electronic Journal of e-Learning, 10(4), 360-367.

Huber, L., \& Watson, C. (2014). Technology: Education and Training Needs of Older Adults. Educational Gerontology, 40(1), 16-25. doi: 10.1080/03601277.2013.768064

Jiménez-Cortés, R. (2004). Las mujeres, las máquinas y la pedagogía de género. En A. Rebollo-Catalán e I. Mercado (Coords.), Mujer y desarrollo: voces para la igualdad (pp. 337-349). Madrid: McGraw-Hill.

Jiménez-Cortés, R. (2015). The influence of informal learning processes of social network sites on the subjective well-being of women in rural areas. Cultura y Educación, 27(2), 407-439. doi: 10.1080/11356405.2015.1034534

Jiménez-Cortés, R., Rebollo-Catalán, A., García-Pérez, R., y Buzón-García, O. (2015). Motivos de uso de las redes sociales virtuales: Análisis de perfiles de mujeres rurales. RELIEVE, 21(1), 1-17. doi: 10.7203/relieve.21.1.5153

Lau, W., \& Yuen, A. (2014). Developing and validating of a perceived ICT literacy scale for junior secondary school students: Pedagogical and educational contributions. Computers \& Education, 78,1-9. doi: 10.1016/j.compedu.2014.04.016

Lee, L., Chen, D., Li, J., \& Lin, T. (2015). Understanding new media literacy: The development of a measuring instrument. Computers \& Education, 85, 84-93. doi: 10.1016/j. compedu.2015.02.006 
Lin, C., Tang, W., \& Kuo, F. (2012). Mommy Wants to Learn the Computer: How Middle-Aged and Elderly Women in Taiwan Learn ICT Through Social Support. Adult Education Quarterly, 62(1), 73-90. doi: $10.1177 / 0741713610392760$

Livingstone, S., \& Helsper, E. (2007). Gradations in digital inclusion: children, young people and the digital divide. New Media \& Society, 9(4), 671696. doi: $10.1177 / 1461444807080335$

Manosevitch, I., \& Tzuk, Y. (2017). Blogging, craft culture, and women empowerment. Cogent Social Sciences, 3, 1-19. doi: 10.1080/23311886.2017.1408753

Meyers, E.M., Erikson, I., \& Small, R.V. (2013). Digital literacy and informal learning environments: An introduction. Learning, Media and Technology, 38(4), 355-367. doi: 10.1080/17439884.2013.783597

MIET (2013). Plan de Inclusión Digital y Empleabilidad. Recuperado de https:// goo.gl/cVXFD1

Nikitov, A., \& Sainty, B. (2014). The role of social media in influencing career success. International Journal of Accounting \& Information Management, 22(4), 273-294. doi: 10.1108/IJAIM-02-2014-0009

Prins, E., Toso, B.W., \& Schafft, K.A. (2009). It Feels Like a Little Family to Me: Social Interaction and Support among Women in Adult Education and Family Literacy. Adult Education Quarterly, 59(4), 335-352. doi: 1177/0741713609331705

Rebollo-Catalán, A., Mayor-Buzón, V., y García-Pérez, R. (2017). Competencias digitales de las mujeres en el uso de las redes sociales virtuales: diferencias según perfil laboral. Revista de
Investigación Educativa, 35(2), 427444. doi: 10.6018/rie.35.2.270881

Rebollo-Catalán, A., y Vico-Bosch, A. (2014). El apoyo social percibido como factor de inclusión digital de las mujeres de entorno rural en las redes sociales virtuales. Comunicar, 43, 173180. doi: 10.3916/C43-2014-17

Rebollo-Catalán, A., Vico-Bosch, A., y García-Pérez, R. (2015). El aprendizaje de las mujeres de las redes sociales y su incidencia en la competencia digital. Prisma Social, 15, 122-146.

Rosenthal, R. (2008). Older ComputerLiterate Women: Their Motivations, Obstacles, and Paths to Success. Educational Gerontology, 34(7), 610626. doi: 10.1080/03601270801949427

Rubio, M.J., y Escofet, A. (2013). Estudio sobre los usos de las TIC y las posibilidades de empoderamiento en las mujeres. Revista Iberoamericana de Educación, 62(3), 1-13.

Sánchez-Vadillo, N., Ortega, O., y VallLlovera, M. (2012). Romper la brecha digital de género. Factores implicados en la opción por una carrera tecnológica. Athenea Digital, 12(3), 115-128. doi: 10.5565/rev/athenead/v12n3.1133

Sloep, P., \& Berlanga, A. (2011). Learning Networks, Networked Learning. Comunicar, 37, 55-63. doi: 10.3916/C37-2011-02-05

Tyner, K., Gutiérrez-Martín, A., y Torrego-González, A. (2015). «Multialfabetización» sin muros en la era de la convergencia. La competencia digital y la cultura del hacer como un revulsivo para una educación continua. Profesorado, 19(2), 42-56.

Vega, L., Vico-Bosch, A., y RebolloCatalán, A. (2015). Motivaciones de uso de las redes sociales para el desarrollo del capital social de las mujeres de 
entorno rural. Icono 14, 13(2), 76-104. doi: 10.7195/ri14.v13i2.839

Vergés, N., Hache, A., y Cruells, E. (2011). Indagando en la relevancia de Internet en el acceso, uso y deseos de las TIC por parte de las mujeres en las TIC. Teoría de la Educación: Educación y Cultura en la Sociedad de la Información, 12(2), 105-121.

Wastiau, P., et al. (2013). The Use of ICT in Education: a survey of schools in Europe. European Journal of
Education, 48(1), 11-27. doi: 10.1111/ ejed. 12020

Wheeler, S. (2015). Learning with 'e's: educational theory and practice in the digital age. Wales: Crown House Publishing.

Yang, C.C., \& Brown, B. (2013). Motives for using facebook, patterns of Facebook activities, and late adolescents. Social adjustment to college. Youth Adolescence, 42, 403416. doi: 10.1007/s10964-012-9836-X 


\section{ANEXO 1}

\section{Escala de aprendizaje en las redes sociales virtuales}

\section{Motivos de aprendizaje}

Aprender a usar las redes sociales e internet me ha servido para:

\begin{tabular}{lcccc}
\hline & Nunca & $\begin{array}{c}\text { Rara } \\
\text { vez }\end{array}$ & $\begin{array}{c}\text { Algunas } \\
\text { veces }\end{array}$ & $\begin{array}{c}\text { Muchas } \\
\text { veces }\end{array}$ \\
\hline $\begin{array}{l}\text { 1. Relacionarme y expresarme más abiertamente } \\
\text { 2. Relacionarme con personas de diversas edades, } \\
\quad \text { culturas, etc. }\end{array}$ & 0 & 1 & 2 & 3 \\
$\begin{array}{l}\text { 3. Reducir la distancia geográfica con personas, } \\
\text { grupos y comunidades }\end{array}$ & 0 & 1 & 2 & 3 \\
$\begin{array}{l}\text { 4. Participar más activamente en la vida cultural } \\
\text { y social de mi comunidad }\end{array}$ & 0 & 1 & 2 & 3 \\
5. Atender mejor mis necesidades y las de mi fa- \\
$\begin{array}{l}\text { milia } \\
\text { 6. Ser más eficiente y productiva en mi trabajo }\end{array}$ & 0 & 1 & 2 & 3 \\
$\begin{array}{l}\text { 7. Experimentar y desarrollar mis hobbies (pin- } \\
\text { tura, cocina, viajes...) }\end{array}$ & 0 & 1 & 2 & 3 \\
$\begin{array}{l}\text { 8. Gestionar mejor mis actividades de formación, } \\
\text { mis estudios }\end{array}$ & 0 & 1 & 2 & 3 \\
$\begin{array}{l}\text { 9. Realizar gestiones administrativas (operacio- } \\
\text { nes bancarias, compra online, firma digital...) }\end{array}$ & 0 & 1 & 2 & 3 \\
10. Promocionar y dar visibilidad a mi empresa & 0 & 1 & 2 & 3 \\
\hline
\end{tabular}




\section{Estrategias de aprendizaje}

Para aprender más sobre internet y redes sociales, prefiero hacerlo:

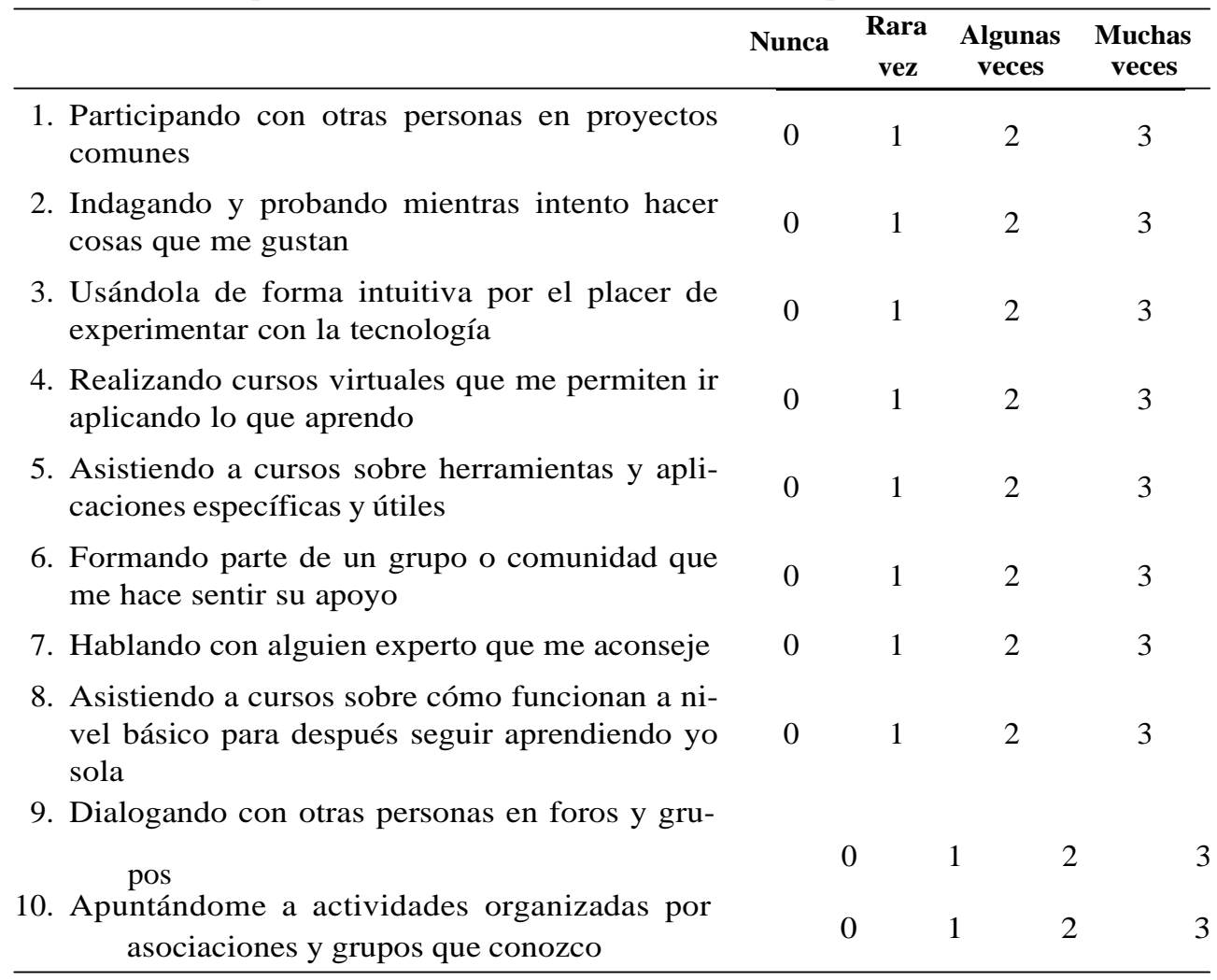

Recursos de aprendizaje 
Cuando he tenido dudas o he querido aprender algo nuevo sobre internet $y$ redes sociales, lo he hecho:

\begin{tabular}{lcccc}
\hline & Nunca & $\begin{array}{c}\text { Rara } \\
\text { vez }\end{array}$ & $\begin{array}{c}\text { Algunas } \\
\text { veces }\end{array}$ & $\begin{array}{c}\text { Muchas } \\
\text { veces }\end{array}$ \\
\hline $\begin{array}{l}\text { 1. Llamando a la línea de ayuda } \\
\text { 2. Pidiendo ayuda a amigos y familiares }\end{array}$ & 0 & 1 & 2 & 3 \\
$\begin{array}{l}\text { 3. Contratando los servicios de un experto } \\
\begin{array}{l}\text { 4. Consultando información por Internet (busca- } \\
\text { dores, blogs...) }\end{array}\end{array}$ & 0 & 1 & 2 & 3 \\
$\begin{array}{l}\text { 5. Buscando vídeos tutoriales por YouTube } \\
\begin{array}{l}\text { 6. Experimentando y probando con la propia tec- } \\
\text { nología }\end{array}\end{array}$ & 0 & 1 & 2 & 3 \\
$\begin{array}{l}\text { 7. Asistiendo a cursos, talleres y otras actividades } \\
\text { formativas presenciales }\end{array}$ & 0 & 1 & 2 & 3 \\
$\begin{array}{l}\text { 8. Participando en foros, grupos y comunidades } \\
\text { virtuales }\end{array}$ & 0 & 1 & 2 & 3 \\
$\begin{array}{l}\text { 9. Realizando cursos virtuales } \\
\begin{array}{l}\text { 10. Observando cómo lo hacen otras personas de } \\
\text { mi entorno }\end{array}\end{array}$ & 0 & 1 & 2 & 3 \\
\hline
\end{tabular}




\section{PERFIL ACADÉMICO Y PROFESIONAL DE LAS AUTORAS}

Alba Vico-Bosch. Contratada predoctoral desde 2014 en el departamento de Métodos de Investigación y Diagnóstico en Educación de la Universidad de Sevilla. Pertenece al grupo de investigación DIME. Obtuvo su título de Máster Universitario en Estudios de Género y Desarrollo Profesional en 2013. Actualmente realiza su tesis doctoral en el marco del proyecto I+D Las mujeres como tejedoras de las redes sociales: estrategias relacionales e inclusión digital, cuya referencia es EDU2013-45134-P.

Ángeles Rebollo-Catalán. Profesora Titular de Universidad del departamento de Métodos de Investigación y Diagnóstico en Educación, Universidad de Sevilla. Su línea de trabajo se vincula al área de los estudios de género en educación y, en especial, al estudio de los procesos de innovación educativa mediados por las tecnologías. Su actividad científica más reciente se vincula a dos proyectos de $\mathrm{I}+\mathrm{D}$ relacionados con el aprendizaje y uso de las mujeres de las redes sociales.

Dirección de las autoras: Universidad de Sevilla

$$
\begin{aligned}
& \text { C/Pirotecnia, s/n } \\
& \text { 41013 - Sevilla } \\
& \text { E-mail: avico@us.es } \\
& \text { rebollo@us.es }
\end{aligned}
$$

Fecha recepción del artículo: 03. Febrero. 2018

Fecha modificación del artículo: 06. Junio. 2018

Fecha aceptación del artículo: 08. Junio. 2018

Fecha revisión para publicación: 20. Junio. 2018 